\title{
Histological evaluation of postnatal retinal development of senescence-accelerated OXYS rats
}

\author{
Darya V. Telegina \\ ICG SB RAS, Novosibirsk, Russia \\ telegina@bionet.nsc.ru
}

\author{
Anna K. Antonenko \\ NSU, Novosibirsk, Russia \\ antonenko-98@bk.ru
}

\author{
Oyuna S. Kozhevnikova \\ ICG SB RAS, Novosibirsk, Russia \\ oidopova@bionet.nsc.ru
}

\begin{abstract}
De novo neurogenesis in the adult mammalian retina very limited. Thereby the structural and functional features formed during the period of maturation and formation of retina can have long-term effects on the further ontogenesis of the tissue, however, the mechanisms of these disorders remain unclear. Using model of premature aging OXYS rats we investigated the early histopathological changes during postnatal retinal neurogenesis. OXYS rats spontaneously develop a retinopathy similar to age-related macular degeneration (AMD). AMD is a complex neurodegenerative disease resulting in a loss of central vision in the elderly. Ganglion, horizontal, and amacrine cells are born in the embryonic phase of rat developmen. Quantitative analysis showed decreasing amacrine cells in OXYS rats as compared Wistar rats (control). At the age of P0 and P1, the number of ganglion and horizontal cells increased in OXYS rats as compared Wistar rats. Bipolar neurons, photoreceptors and Müller glia are born postnatally. We did not find changes in Muller cells. The number of photoreceptor's nuclei per column in Wistar rats increased at the age of P10 and decreased at the age of P14. In OXYS rats, maximum of number of nuclei per column accounted for age of P14 and then decreased. The number of rod bipolar neurons gradually increased by age of P14 in Wistar rats and P10 in OXYS rats. Our results indicating an alteration of retinal formation in OXYS rats during the postnatal period, which may contribute to the early development of their signs of AMD.
\end{abstract} rats

Keywords - neurogenesis, retina, AMD, OXYS rats, Wistar

\section{Introduction}

The retina is a delicate neural tissue responsible for light signal capturing, modulating, and passing to mid-brain. The adult vertebrate retina comprises six major cell classes derived from a common progenitor cell, only one of which, the Müller cell, is a glial cell. It is important that the mammalian neural retina, however, like many other regions of the central nervous system, lacks any significant regenerative potential to replace lost neurons after retinogenesis is complete. Consequently, any damage leading to the death of the light sensing photoreceptor cells or their support cells, either through injury or disease, typically leads to permanent visual impairment. But our understanding of human eye development is very limited, especially the contribution of impaired retinogenesis in development of retinal degeneration.

The retinal neurons include cone and rod photoreceptors and horizontal, amacrine, bipolar, and ganglion cells. There is evidence to suggest that ganglion, horizontal, amacrine, and early-born cone photoreceptor cells originate froma common pool of intermediate progenitors (EBPs). Bipolar, Müller, and late-born photoreceptor cells share a pool of common intermediate progenitors (LBPs) [1]. In rat and mouse EBPs arise from E10 to P4 and peak at E16, while
LBPs appear from E13 to P10 and peak at P2. Retinogenesis is complete at $\mathrm{P} 20$ in rodents.

Understanding the programs underlying the developmental process not only will provide insights into how each individual cell type is formed and what function it may have, but also will give clues on how to treat retinal diseases in the future such as age-related macular degeneration (AMD). AMD is a complex neurodegenerative disease resulting in a loss of central vision in the elderly. Here, we used a suitable genetic experimental model of AMD is senescence-accelerated OXYS rats, which spontaneously develop a phenotype similar to human age-related disorders including AMD-like retinopathy. Retinopathy that develops in OXYS rats already at a young age corresponds (in terms of clinical manifestations and morphological characteristics) to the dry atrophic form of AMD in humans. Nonetheless, neovascularization develops in some $(\sim 10-20 \%)$ of these rats with age. The clinical signs of AMD-like retinopathy appear by the age of 3 months in $100 \%$ of OXYS rats against the background of a reduction in the transverse area of the RPE, impairment of choroidal microcirculation, and retinal thinning. The progression of these abnormalities in OXYS rats until the age of 10 months is accompanied by a significant reduction in thickness of the photoreceptor cell layer and a decrease in the number of photoreceptor cell nuclei of the outer nuclear layer, especially in the central part of the retina [2].

We aimed to give a detailed qualitative and quantitative description on the early histopathological changes in the postnatal development retina of OXYS and Wistar rats (as control) and compare our results with previous studies dealing with the senescence-accelerated OXYS rats.

\section{Methods}

All procedures of the present study were performed in concordance with the ARVO statement for the Use of Animals in Ophthalmic and Vision Research and were approved the Institutional Review Board of the Institute of Cytology and Genetics, according to The Guidelines for Manipulations of Experimental Animals. The study was carried out on OXYS and Wistar (control) rats in the critical days of postnatal retinal neurogenesis: $0,1,3,5,7$, $10,14,20$. We used standard techniques of immunohistochemistry and TUNEL assay [3].

\section{Results}

By immunohistochemistry using the specific antibodies carried out a comprehensive assessment of cell populations differentiating in the postnatal period into photoreceptors, Müller cells, amacrine, ganglion, bipolar and horizontal neurons and also evaluated the dynamics of apoptosis using the TUNEL assay. 
Müller glia cells were analysed using antibodies against cell type specific cytoskeletal elements: vimentin and nestin. Nestin is an intermediate filament protein that is known as a neural stem/progenitor cell marker. It is expressed in undifferentiated CNS cells during development. Vimentin immunoreactivity throughout the whole depth of the retina of OXYS and Wistar rats was maximum in P0 and was gradually decreased with age. Double labelling with anti - nestin revealed a similar pattern of immunoreactivity in radial cell processes throughout the retina, which colocalized with vimentin immunoreactivity. Comparing sections of OXYS and Wistar rats during postnatal retinal development, we did not find any qualitative or significant quantitative changes in Vimentin and Nestin labelling.

Recoverin is a protein in rods that regulates rhodopsin phosphorylation in visual transduction and enhances visual sensitivity. In the OXYS and Wistar retinas, strong recoverin immunoreactivity was detected in putative differentiating photoreceptors, localized to the apical surface of the neuroblast layer (NBL) and in sparse cells found deeper in the inner NBL (bipolar cells). Recoverin staining was seen throughout the developing outer nuclear layer (ONL) in rat retina. In the ONL, nuclei are typically arranged in columns on well oriented sections. Recoverinlabelled nuclei formed in well-organized columns starting on the fifth postnatal day in OXYS and Wistar rats. Therefore, we counted the average number of recoverinlabelled nuclei per column. In Wistar rat's retina the recoverin-labelled layer was well-formed at the age of P5 whereas in OXYS rat's retina - at the age of P7. Quantitative analysis showed increasing the number of nuclei per column in Wistar rats at the age of P10 and decreased at the age of P14. In OXYS rats, we observed a similar trend but the period time was altered. Therefore, the maximum of number of nuclei per column accounted for age of P14 in OXYS rats and then the number of nuclei per column decreased (at the age of P20). It is important that average number of recoverin-labelled nuclei per column was greater in Wistar rats than OXYS rats at the age of P5 and P7. However, this parameter was greater in OXYS rats than Wistar rats at the age of P10, P14 and P20. Besides the photoreceptors both the ON and OFF cone bipolar cells can be labelled with recoverin antibody. Average number of recoverin-labelled bipolar neurons gradually increased by age of P14 in Wistar rats. In OXYS rats this parameter was drammaticaly increased by age of P10 and then decreased. We proposed that this phenomenon associated with adaptive mechanisms.

Parvalbumin stains dominantly AII amacrine cells, along with a few wide field amacrine cells, a population of bipolar cells and a few cells in the ganglion cell layer [4]. The AII amacrine cells is one well-characterized subset of glycinergic amacrine cells [5]. Regarding AII positive amacrine cell populations, we detected a weakly staining intensity for parvalbumin labelled cells before P3 in OXYS and Wistar rats. After P3 parvalbumin labelled cells are characterized by strongly immunoreactivity and distinctly morphology in both rat strains. Quantitative analysis showed decreasing parvalbumin labelled cells in OXYS rats as compared Wistar rats at the all ages (P0-P20).

The antibody against calbindin, a calcium binding protein involved in calcium transport, stains horizontal cells and a few cells in the INL and GCL under control conditions [4]. We observed a weakly staining intensity for calbindin labelled cells before P5 in OXYS and Wistar rats. After P5 calbindin labelled cells are characterized by strongly immunoreactivity and distinctly morphology in both rat strains. In Wistar rats we detected no significant difference in horizontal cells at the all ages. In OXYS rats average number of calbindin labelled cells was greater that than in Wistar rats at the age of $\mathrm{P} 0$. At the age of $\mathrm{P} 1$, number og horizontal neurons dramatically decreased. After P0 significant difference between OXYS and Wistar rats is not observe.

In next step we used anti-NeuN antibody as a marker for ganglion cells. Our results showed that NeuN was strongly expressed in ganglion and displaced amacrine cells in the GCL and weakly expressed in amacrine cells and horizontal cells in the INL in OXYS and Wistar rats. The average number of NeuN-positive cells decreased in the age of P7 in Wistar and OXYS rats. We detected increasing number of NeuN labelled ganglion cells in OXYS rats at the age of $\mathrm{P} 0$ and $\mathrm{P} 1$ as compared Wistar rats.

A TUNEL analysis of retinal cryosections showed a higher apoptosis activity (especcialy P7, P10 and P14 days) in OXYS rats, which indicates an enhanced reduction of neurons.

Summary, we obtained the first results indicating a alteration of retinal formation in OXYS rats during the postnatal period, which may contribute to the early development of their signs of AMD.

\section{Conclusion}

Thus, using senescence-accelerated OXYS rats, which spontaneously develop AMD-like retinopathy, we obtained the first results of a study of the contribution of changes in neurogenesis in the retina during the postnatal period to the early development of AMD-like retinopathy in OXYS rats. We hypothesize that the histological features presented in OXYS rats during postnatal neurogenesis could underlie the functional and structural alterations and can have long-term effects on the further ontogenesis of the tissue.

\section{ACKNOWLEDGMENT}

This study was supported by the Russian Foundation for Basic Research (18-015-00320) and by a Russian Scientific Foundation Grant (19-15-00044).

\section{REFERENCES}

[1] Jin, K. (2017). Transitional progenitors during vertebrate retinogenesis. Molecular neurobiology, 54(5), 3565-3576. https://doi.org/10.1007/s12035-016-9899-x

[2] Telegina, D., Kozhevnikova, O., Bayborodin, S. et al. (2017). Contributions of age-related alterations of the retinal pigment epithelium and of glia to the AMD-like pathology in OXYS rats. Sci Rep, 7, 41533. https://doi.org/10.1038/srep41533

[3] Kozhevnikova, O. S., Telegina, D. V., Tyumentsev, M. A., Kolosova, N. G. (2019). Disruptions of Autophagy in the Rat Retina with Age During the Development of Age-Related-MacularDegeneration-like Retinopathy. International Journal of Molecular Sciences, 20(19), 4804. https://doi.org/10.3390/ijms20194804

[4] Szabó, K., et al. (2017). Histological evaluation of diabetic neurodegeneration in the retina of Zucker Diabetic Fatty (ZDF) rats. Scientific reports, 7(1), 1-17. https://doi.org/10.1038/s41598017-09068-6

[5] Balasubramanian, R., \& Gan, L. (2014). Development of retinal amacrine cells and their dendritic stratification. Current $\begin{array}{lll}\text { ophthalmology } \quad \text { reports, } & \text { 2(3), }\end{array}$ https://doi.org/10.1007/s40135-014-0048-2 